\title{
Impact of Surgery Type on Quality of Life in Breast Cancer Patients
}

\author{
Mustafa Akça ${ }^{2}$, Alper Ata ${ }^{1}$, Erdinç Nayır ${ }^{3}$, Süleyman Erdoğdu ${ }^{3}$, Ali Arıcan ${ }^{3}$ \\ ${ }^{1}$ Clinic of Medical Oncology, Mersin State Hospital, Mersin, Turkey \\ ${ }^{2}$ Department of Internal Medicine, Mersin University, Faculty of Medicine, Mersin, Turkey \\ ${ }^{3}$ Department of Medical Oncology, Mersin University, Faculty of Medicine, Mersin, Turkey
}

\section{ABSTRACT}

Objective: Breast cancer can lead to alterations in quality of life of the patients. The aim of this study is to evaluate the changes in quality of life of the female patients who had undergone surgical treatment for breast cancer.

Materials and Methods: A total of 250 female patients (breast-preserving surgery (BPS), n=27, 11\%; modified radical mastectomy (MRM), $n=194$, $77 \%$, and simple mastectomy $(\mathrm{SM}), \mathrm{n}=29 ; 11 \%)$ aged between $28-55$ years (47.4 $6.4 \mathrm{yrs})$ were included in the study. Patient information, demographic characteristics, income, and treatment modalities applied were recorded. Validated Turkish versions of EORTC QLQ-C30, and EORTC-BR23 questionnaires were used for all patients.

Results: Breast-preserving surgery has a more favorable impact on general well-being, physical role, cognitive, psychological, and social functions, and symptom scale scores. When the identical parameters were taken into consideration, relatively favorable outcomes of BPS on the patients were observed relative to mastectomized patients. Besides, though not statistically significant, BPS has more patient-friendly effects on sexual function and sexual satisfaction in comparison with mastectomy. Patients with advanced stage disease and elder patients had more unfavorable health related quality of life (HRQoL) scores than younger patients, and those in their early stages of breast cancer.

Conclusion: Quality of life of BPS patients is less adversely affected relative to mastectomized patients. In the decision-making process, quality of life should be taken into consideration.

Key words: Breast cancer, mastectomy, quality of life, sexual activity, psychosocial factors

\section{Introduction}

Breast cancer is the most frequently seen cancer among women, and breast cancer-related deaths rank third among all cancer deaths (1). Operations for breast cancer disfiguring body image or impairing functions of body parts, and especially surgical interventions aiming at facial, and mammary regions have profound psychological effects on women's body image, quality of life, and sexual life (2). Anxiety, depression, anger, guiltiness, fear, and social isolation are the mostly encountered post-mastectomy problems (2).

World Health Organization (WHO) defines quality of life as 'individuals' perception of their position in life within the whole context of the culture and value systems of the social environment in which they live and in relation to their goals, expectations, standards and concerns 3).

Many indicators should be considered in the measurement of quality of life. Among them, 'functional capacity' indicates the ability to perform daily living activities, and the level of physical activity. "Social function" reflects the ability of an individual to interact with other members of his/her family, friends, and other people, while "emotional function" reflects the level of anxiety induced by side effects developed as a result of treatments applied, and disease-related symptoms such as pain (4).

Cancer can be defined as a chronic disease that involves physical inadequacies, and psychological problems with periods of remissions, and exacerbations, and creates difficulties in maintaining short and long-term compliance (5). Quality of life is one of the important concepts not to be forgotten for cancer patients during their lives, and throughout their curative and/or palliative treatments.

This study was presented at the $4^{\text {th }}$ Conference on Medical Oncology. 21-25 March 2012, Antalya, Turkey. Address for Correspondence: 
In parallel with the advances in the treatment of breast cancer with their favorable effects on its incidence, and survival rates, detailed comprehension of the problems encountered by patients during their treatment, disease, and remissions portends critical importance.

Various questionnaire forms have been used in oncology to assess quality of life of cancer patients. European Organization for Research and Treatment of Cancer (EORTC) QLQ-C30 questionnaire forms contain 30 items which can be applied on every type of cancer. EORTC QLQ-BR23 questionnaire form has been designed especially for breast cancer patients, and contains 23 items. The participants mark one answer among four alternatives (1-not at all; 2-a little, 3- quite a bit 4-very much) (6-8).

The aim of this study is to investigate the changes in the quality of life, and social, psychological, and sexual life of the patients who had undergone breast surgery for breast cancer using EORTC QLQC30, and EORTC QLQ-BR23 questionnaire forms.

\section{Materials and Methods}

Breast cancer patients treated, and followed-up by the Mersin University department of medical oncology, the years 2000, and 2010 were investigated. The female patients who survived after modified radical or simple mastectomy or breast-preserving surgery, and volunteered to fill the consent forms were included in the study. The study participants were also literate, married or had a sexual partner without any concomitant medical, and/or psychological disorder that impair(s) mental and/or bodily functions of the patients. Patients who had not undergone primary breast cancer surgery, those with metastatic breast cancer or illiterate cases were not included in the study. Ethnic and geographic differences were not taken into consideration for all study participants. The patients' files were retrospectively analyzed, and patient-related data (information concerning age, address of correspondence, social, and demographic characteristics, income, and educational levels, menopausal state, grade, and stage of the tumor, adjuvant and/or neoadjuvant surgical, and medical treatments) were recorded.

The patients were categorized into 3 groups based on their ages as follows: Group 1; 28-35 years; Group 2: 36-45 years, and Group 3: 46-55 years (Table 1 ). The patients were interrogated during outpatient control visits. After obtaining their volunteer consent forms, the questionnaire forms were filled up.

In our investigation, Turkish version (v.3.0) of the mostly preferred EORTC QLQ-C30 and EORTC QLQ-BR23 questionnaire forms were used.

Mersin University Ethic Committee approved our study in 03.11.2010 by number: 71. Each patient signed detailed informed consent forms.

\section{Statistical Analysis}

One-way analysis of variance was used in comparisons between subgroups as for the variables as age groups, stage of the cancer, and type of the operation performed based on total subscale scores. For pair wise comparisons between groups demonstrating differences, Tukey HSD test was used. Cronbach's alpha coefficient was calculated to assess internal consistency of scale items. The test was deemed valid

\section{Table 1. Demographic characteristics of the patients}

\begin{tabular}{|c|c|}
\hline \multicolumn{2}{|l|}{ Age (yrs), (n, \%) } \\
\hline All groups (mean $\pm S D ;$ range) & $47.4 \pm 6.4$ (28-55 years) \\
\hline Group 1 (28-35 years) (n, \%) & $19(7.6 \%)$ \\
\hline Group 2 ( $36-45$ years) $(n, \%)$ & $69(27.6 \%)$ \\
\hline Group 3 (46-55 years) (n, \%) & $162(64.8 \%)$ \\
\hline \multicolumn{2}{|l|}{ Marital status, (n, \%) } \\
\hline Married & $237(94.8 \%)$ \\
\hline Single & $4(1.6 \%)$ \\
\hline Divorced & $9(3.6 \%)$ \\
\hline \multicolumn{2}{|l|}{ Educational level, (n, \%) } \\
\hline Primary school & $171(68.4 \%)$ \\
\hline Lycée & $66(26.4 \%)$ \\
\hline High school, university & $13(5.2 \%)$ \\
\hline \multicolumn{2}{|l|}{ Occupation, (n, \%) } \\
\hline Unemployed & $213(85.2)$ \\
\hline Employed & $37(14.8)$ \\
\hline \multicolumn{2}{|c|}{ Income level (monthly wages), (n, \%) } \\
\hline$A(<750 \mathrm{TL})$ & 49 (19.6) \\
\hline B (750-1500 TL) & $151(60.4)$ \\
\hline$C(>1500 \mathrm{TL})$ & $50(20.0)$ \\
\hline \multicolumn{2}{|l|}{ Living Environment, (n, \%) } \\
\hline Urban & $178(71.2 \%)$ \\
\hline Rural & $72(28.8 \%)$ \\
\hline
\end{tabular}

and reliable when a Cronbach coefficient of $\geq 0.70$ was obtained. In all comparisons performed, type I error rate was determined as 0.05 . In all comparisons, $\mathrm{p}$ values calculated below this level were considered significant. All analyses were realized using SPSS for Windows 11.5.1 statistical (SPSS Inc., USA) program.

\section{Results}

A total of 250 eligible female patients who were followed up by the Mersin University department of medical oncology, and volunteered to participate in the survey were included in the study. Mean age of the study participants was $47.4 \pm 6.4$ (28-55 yrs) years. Demographic data were summarized in Table 1 .

Our patients had difficulty in answering the questions inquiring their libido, and income level. Questions related to sexual life, sexual pleasure, and income level were not answered by $5.2(\mathrm{n}=7)$, and 2.8 $(\mathrm{n}=7)$ percent of the patients, respectively.

Patients who had undergone breast conserving surgery (BCS), and simple mastectomy (SM) had more favorable outcomes relative to modified radical mastectomy (MRM) patients as assessed by EORTC QLQ-C30 questionnaire scales of social functioning $(\mathrm{p}<0.01)$, fatigue $(\mathrm{p}=0.02)$, constipation $(\mathrm{p}<0.01)$, and global quality of life $(\mathrm{p}<0.001)$. As for other functional scales as physical $(\mathrm{p}=0.2)$, cognitive $(\mathrm{p}=0.6)$, emotional $(\mathrm{p}=0.3)$, and role $(\mathrm{p}=0.3)$ functioning, MRM resulted in worse outcomes without any statistically significant difference from other breast cancer treatment modalities. Re- 
Table 2. Comparison of types of surgery based on EORTC QLQ-C30 scale scores

\begin{tabular}{|c|c|c|c|c|}
\hline \multirow[b]{2}{*}{ EORTC QLQ-C30 } & $\begin{array}{c}\text { MRM } \\
\text { n: } 194\end{array}$ & $\begin{array}{c}\text { SM } \\
\mathrm{n}: 29\end{array}$ & $\begin{array}{l}\text { BPS } \\
\mathrm{n}: 27\end{array}$ & \multirow[b]{2}{*}{$\begin{array}{c}\text { P } \\
\text { value }\end{array}$} \\
\hline & $\begin{array}{c}\text { Mean士 } \\
\text { SD }\end{array}$ & $\begin{array}{c}\text { Mean士 } \\
\text { SD }\end{array}$ & $\begin{array}{c}\text { Mean士 } \\
\text { SD }\end{array}$ & \\
\hline \multicolumn{5}{|l|}{ GLOBAL HEALTH STATE } \\
\hline General Well-being & $8.7 \pm 2.7$ & $9.8 \pm 2.5$ & $11.5 \pm 1.7$ & $<0.001$ \\
\hline \multicolumn{5}{|l|}{ FUNCTIONAL SCALES } \\
\hline Physical functioning & $8.9 \pm 3.0$ & $9.4 \pm 2.9$ & $8.0 \pm 1.5$ & 0.2 \\
\hline Role functioning & $3.7 \pm 1.5$ & $3.6 \pm 1.6$ & $3.2 \pm 1.0$ & 0.3 \\
\hline Cognitive functioning & $3.6 \pm 1.5$ & $3.5 \pm 1.4$ & $3.3 \pm 1.0$ & 0.6 \\
\hline Emotional functioning & $8.7 \pm 3.2$ & $8.0 \pm 2.9$ & $7.1 \pm 1.8$ & 0.3 \\
\hline Social functioning & $4.6 \pm 1.8$ & $3.9 \pm 1.8$ & $3.3 \pm 1.1$ & $<0.01$ \\
\hline \multicolumn{5}{|l|}{ SYMPTOM SCALES } \\
\hline Dyspnea & $1.5 \pm 0.7$ & $1.6 \pm 0.6$ & $1.3 \pm 0.5$ & 0.3 \\
\hline Nausea-vomiting & $2.9 \pm 1.3$ & $2.8 \pm 1.5$ & $2.7 \pm 0.9$ & 0.7 \\
\hline Loss of appetite & $1.6 \pm 0.8$ & $1.7 \pm 0.7$ & $1.4 \pm 0.5$ & 0.2 \\
\hline Sleep disorders & $2.2 \pm 1.0$ & $2.3 \pm 0.9$ & $1.8 \pm 0.6$ & 0.2 \\
\hline Pain & $3.8 \pm 1.5$ & $3.6 \pm 1.2$ & $3.5 \pm 0.9$ & 0.4 \\
\hline Fatigue & $6.5 \pm 2.3$ & $6.2 \pm 2.2$ & $5.2 \pm 1.0$ & 0.02 \\
\hline Constipation & $1.6 \pm 0.8$ & $2.0 \pm 1.0$ & $1.5 \pm 0.6$ & 0.01 \\
\hline Diarrhea & $1.3 \pm 0.6$ & $1.2 \pm 0.4$ & $1.25 \pm 0.4$ & 0.6 \\
\hline Financial difficulties & $2.5 \pm 1.0$ & $2.3 \pm 1.0$ & $2.2 \pm 0.9$ & 0.3 \\
\hline \multicolumn{5}{|c|}{$\begin{array}{l}\text { BPS: Breast preserving surgery; EORTC-QLQ: European Organization for } \\
\text { Research and Treatment of Cancer, quality of life questionnaire; } \\
\text { MRM: Modified radical mastectomy; SM: Simple mastectomy, } \\
\text { SD: Standard deviation }\end{array}$} \\
\hline
\end{tabular}

garding these parameters, any statistically significant difference was not detected between SM, and BCS groups (Table 2, and Table 3).

Evaluations based on EORTC QLQ-BR23 scale scores have revealed that when compared with MRM patients, body image $(\mathrm{p}<0.001)$, future perspectives $(\mathrm{p}<0.001)$, arm symptoms $(\mathrm{p}=0.05)$, and hair loss $(\mathrm{p}<0.006)$ scores were more favorable in BCS, and SM patients. Three surgical procedures did not differ as for subgroups of sexual life, sexual pleasure, adverse effects of the treatments used, and breast symptoms ( $>>0.05)$ (Table 4 and Table 5).

When responses to EORTC QLQ-C30 questions were evaluated, and categorized according to age groups, alterations in cognitive functioning were relatively significant $(\mathrm{p}=0.02)$ in Group 3 without any significant intergroup difference as for other domains of the scale (Table 6). When responses given to EORTC QLQ-BR23 questionnaire items were evaluated based on age groups in Group 1, parameters of sexual life, and sexual pleasure, and in Group 2, those of body image, future perspectives, adverse effects of drugs, breast and arm symptoms, and hair loss were relatively more unfavorable. Group 3 had more favorable outcomes without any statistically significant
Table 3. Comparison of mastectomy, and BPS based on EORTC QLQ-C30 scale scores

\begin{tabular}{|c|c|c|c|}
\hline \multirow[b]{2}{*}{ EORTC QLQ-C30 } & $\begin{array}{c}\text { MASTECTOMY } \\
\text { n: } 223\end{array}$ & $\begin{array}{l}\text { BPS } \\
\mathrm{n}: 27\end{array}$ & \multirow{2}{*}{$\begin{array}{c}P \\
\text { value }\end{array}$} \\
\hline & Mean士SD & Mean士SD & \\
\hline \multicolumn{4}{|l|}{ GLOBAL HEALTH STATE } \\
\hline General well-being & $8.4 \pm 2.5$ & $11.8 \pm 19$ & 0.002 \\
\hline \multicolumn{4}{|l|}{ FUNCTIONAL SCALES } \\
\hline Physical functioning & $9.0 \pm 3.0$ & $8.0 \pm 1.5$ & 0.3 \\
\hline Role functioning & $3.6 \pm 1.5$ & $3.2 \pm 1.0$ & 0.2 \\
\hline Cognitive functioning & $3.6 \pm 1.5$ & $3.3 \pm 1.0$ & 0.7 \\
\hline Emotional functioning & $8.6 \pm 3.2$ & $7.1 \pm 1.8$ & 0.07 \\
\hline Social functioning & $4.5 \pm 1.8$ & $3.3 \pm 1.1$ & 0.002 \\
\hline \multicolumn{4}{|l|}{ SYMPTOM SCALES } \\
\hline Dyspnea & $1.5 \pm 0.7$ & $1.3 \pm 0.5$ & 0.25 \\
\hline Nausea-vomiting & $2.9 \pm 1.3$ & $2.7 \pm 0.9$ & 0.82 \\
\hline Loss of appetite & $8.9 \pm 2.7$ & $11.0 \pm 1.8$ & 0.001 \\
\hline Sleep disorders & $2.2 \pm 0.9$ & $1.8 \pm 0.6$ & 0.14 \\
\hline Pain & $3.8 \pm 1.5$ & $3.5 \pm 0.9$ & 0.46 \\
\hline Fatigue & $6.5 \pm 2.3$ & $5.2 \pm 1.0$ & 0.007 \\
\hline Constipation & $1.6 \pm 0.9$ & 1.4.0.6 & 0.62 \\
\hline Diarrhea & $1.7 \pm 0.8$ & $1.6 \pm 0.8$ & 0.4 \\
\hline Financial difficulties & $2.4 \pm 1.0$ & $2.2 \pm 0.9$ & 0.2 \\
\hline
\end{tabular}

Table 4. Comparison of surgical treatment alternatives based on EORTC QLQ-BR23 scale scores

\section{EORTC QLQ-BR23}

\begin{tabular}{|c|c|c|}
\hline $\begin{array}{l}\text { MRM } \\
\text { n: } 194\end{array}$ & $\begin{array}{c}\text { SM } \\
n: 29\end{array}$ & $\begin{array}{c}\text { BPS } \\
\text { n: } 27\end{array}$ \\
\hline $\begin{array}{c}\text { Mean } \pm \\
\text { SD }\end{array}$ & $\begin{array}{c}\text { Mean } \pm \\
\text { SD }\end{array}$ & $\begin{array}{c}\text { Meant } \\
\text { SD }\end{array}$ \\
\hline
\end{tabular}

FUNCTIONAL SCALES

$\begin{array}{lcccc}\text { Body image } & 10.6 \pm 3.7 & 8.5 \pm 3.5 & 7.4 \pm 2.2 & <0.001 \\ \text { Sexual life } & 3.1 \pm 1.2 & 3.0 \pm 1.1 & 2.8 \pm 1.0 & 0.45 \\ \text { Sexual pleasure } & 1.6 \pm 0.7 & 1.5 \pm 0.7 & 1.4 \pm 0.6 & 0.5 \\ \text { Future perspectives } & 2.7 \pm 1.1 & 2.1 \pm 0.9 & 2.0 \pm 0.7 & <0.001 \\ \text { SYMPTOM SCALES } & & & & \\ \text { Adverse effects } & 13.9 \pm 4.4 & 13.8 \pm 3.8 & 12.1 \pm 2.7 & 0.1 \\ \text { Breast symptoms } & 6.8 \pm 2.4 & 6.7 \pm 2.3 & 5.8 \pm 1.7 & 0.1 \\ \text { Arm symptoms } & 5.9 \pm 2.0 & 5.5 \pm 2.0 & 5.0 \pm 1.2 & 0.05 \\ \text { Hair loss } & 2.5 \pm 1.1 & 2.4 \pm 1.1 & 1.8 \pm 0.5 & 0.006\end{array}$

BPS: Breast preserving surgery; EORTC-QLQ: European Organization for Research and Treatment of Cancer quality of life questionnaire; MRM: Modified radical mastectomy; SM: Simple mastectomy, SD: Standard deviation

Responses given to EORTC QLQ-C30 survey questions were evaluated based on the stages of the disease; generally, stage 1 patients had more favorable quality of life parameters relative to other patients in stage 2 , and 3 . As for subgroups of diarrhea $(p<0.05)$, and fi- 
Table 5. Comparison of mastectomy, and BPS based on EORTC QLQ-BR23 scale scores

\begin{tabular}{|c|c|c|c|}
\hline \multirow[b]{2}{*}{ EORTC QLQ-BR23 } & $\begin{array}{c}\text { MASTECTOMY } \\
\text { n: } 223\end{array}$ & $\begin{array}{l}\text { BPS } \\
\text { n: } 27\end{array}$ & \multirow{2}{*}{$\begin{array}{c}P \\
\text { value }\end{array}$} \\
\hline & Mean士SD & MeantSD & \\
\hline \multicolumn{4}{|l|}{ FUNCTIONAL SCALES } \\
\hline Body image & $10.3 \pm 3.7$ & $7.4 \pm 2.2$ & 0.001 \\
\hline Sexual life & $3.1 \pm 1.2$ & $2.8 \pm 1.0$ & 0.25 \\
\hline Sexual pleasure & $1.6 \pm 0.7$ & $1.4 \pm 0.6$ & 0.25 \\
\hline Future perspectives & $2.6 \pm 1.1$ & $2.0 \pm 0.6$ & 0.002 \\
\hline \multicolumn{4}{|l|}{ SYMPTOM SCALES } \\
\hline Adverse effects & $13.9 \pm 4.3$ & $12.1 \pm 2.8$ & 0.047 \\
\hline Breast symptoms & $6.8 \pm 2.4$ & $5.8 \pm 1.7$ & 0.03 \\
\hline Arm symptoms & $5.9 \pm 2.0$ & $5.0 \pm 1.2$ & 0.04 \\
\hline Hair loss & $2.5 \pm 1.1$ & $1.8 \pm 0.5$ & 0.002 \\
\hline
\end{tabular}

Table 6. Comparison of age groups based on EORTC QLQ-C30 scale scores

\begin{tabular}{|c|c|c|c|c|}
\hline EORTC QLQ-C30 & $\begin{array}{c}\text { Group } 1 \\
\text { n: } 19 \\
\text { Mean士 } \\
\text { SD }\end{array}$ & $\begin{array}{c}\text { Group } 2 \\
\text { n: } 69 \\
\text { Mean士 } \\
\text { SD }\end{array}$ & $\begin{array}{c}\text { Group } 3 \\
\text { n: } 162 \\
\text { Mean士 } \\
\text { SD }\end{array}$ & $\begin{array}{c}P \\
\text { value }\end{array}$ \\
\hline \multicolumn{5}{|l|}{ GLOBAL HEALTH STATE } \\
\hline General well-being & $9.2 \pm 2.1$ & $8.6 \pm 2.8$ & $8.9 \pm 2.7$ & 0.1 \\
\hline \multicolumn{5}{|l|}{ FUNCTIONAL SCALES } \\
\hline Physical functioning & $8.8 \pm 3.3$ & $9.2 \pm 2.9$ & $8.8 \pm 2.8$ & 0.6 \\
\hline Role functioning & $3.0 \pm 1.1$ & $3.8 \pm 1.5$ & $3.6 \pm 1.5$ & 0.09 \\
\hline Cognitive functioning & $3.6 \pm 1.5$ & $3.9 \pm 1.6$ & $3.4 \pm 1.4$ & 0.02 \\
\hline Emotional functioning & $8.1 \pm 3.4$ & $8.9 \pm 3.2$ & $8.3 \pm 3.1$ & 0.4 \\
\hline Social functioning & $4.4 \pm 1.8$ & $4.6 \pm 1.9$ & $4.3 \pm 1.8$ & 0.4 \\
\hline \multicolumn{5}{|l|}{ SYMPTOM SCALES } \\
\hline Dyspnea & $1.5 \pm 0.7$ & $1.6 \pm 0.7$ & $1.4 \pm 0.6$ & 0.3 \\
\hline Nausea-vomiting & $2.4 \pm 0.7$ & $2.9 \pm 1.3$ & $3.0 \pm 1.4$ & 0.3 \\
\hline Loss of appetite & $1.5 \pm 0.8$ & $1.6 \pm 0.7$ & $1.6 \pm 0.8$ & 0.6 \\
\hline Sleep disorders & $2.0 \pm 0.9$ & $2.2 \pm 0.9$ & $2.1 \pm 0.9$ & 0.6 \\
\hline Pain & $4.1 \pm 1.5$ & $4.0 \pm 1.4$ & $3.7 \pm 1.4$ & 0.2 \\
\hline Fatigue & $5.8 \pm 1.8$ & $6.7 \pm 2.2$ & $6.2 \pm 2.3$ & 0.2 \\
\hline Constipation & $1.4 \pm 0.7$ & $1.8 \pm 0.9$ & $1.6 \pm 0.8$ & 0.2 \\
\hline Diarrhea & $1.2 \pm 0.4$ & $1.3 \pm 0.6$ & $1.2 \pm 0.5$ & 0.7 \\
\hline Financial difficulties & $2.8 \pm 0.9$ & $2.5 \pm 1.1$ & $2.3 \pm 1.0$ & 0.009 \\
\hline
\end{tabular}

nancial difficulties $(\mathrm{p}<0.001)$ a statistically significant difference was observed, while differences between other subgroups were not statistically significant ( $p>0.05$ ) (Table 8). Assessments based on responses given to EORTC QLQ-BR23 questionnaire items demonstrated im-
Table 7. Evaluation of age groups based on EORTC QLQ-BR23 scale scores

\begin{tabular}{|c|c|c|c|c|}
\hline \multirow[b]{2}{*}{ EORTC QLQ-BR23 } & $\begin{array}{c}\text { Group } 1 \\
\text { n: } 19\end{array}$ & $\begin{array}{c}\text { Group } 2 \\
\text { n: } 69\end{array}$ & $\begin{array}{c}\text { Group } 3 \\
\text { n: } 162\end{array}$ & \multirow[b]{2}{*}{$\begin{array}{c}\text { P } \\
\text { value }\end{array}$} \\
\hline & $\begin{array}{l}\text { Mean士 } \\
\text { SD }\end{array}$ & $\begin{array}{c}\text { Meant } \\
\text { SD }\end{array}$ & $\begin{array}{l}\text { Mean } \pm \\
\text { SD }\end{array}$ & \\
\hline \multicolumn{5}{|l|}{ FUNCTIONAL SCALES } \\
\hline Body image & $10.3 \pm 3.4$ & $10.7 \pm 3.7$ & $9.7 \pm 3.8$ & 0.2 \\
\hline Sexual life & $3.3 \pm 1.2$ & $3.0 \pm 1.1$ & $3.1 \pm 1.2$ & 0.3 \\
\hline Sexual pleasure & $1.7 \pm 0.9$ & $1.5 \pm 0.6$ & $1.6 \pm 0.7$ & 0.4 \\
\hline Future perspectives & $2.6 \pm 1.0$ & $2.8 \pm 1.1$ & $2.5 \pm 1.1$ & 0.25 \\
\hline \multicolumn{5}{|l|}{ SYMPTOM SCALES } \\
\hline Adverse effects & $12.8 \pm 4.4$ & $14.8 \pm 4.1$ & $13.4 \pm 4.1$ & 0.06 \\
\hline Breast symptoms & $6.6 \pm 2.4$ & $7.3 \pm 2.3$ & $6.5 \pm 2.4$ & 0.06 \\
\hline Arm symptoms & $5.6 \pm 2.4$ & $6.1 \pm 2.0$ & $5.6 \pm 1.9$ & 0.2 \\
\hline Hair loss & $2.4 \pm 1.2$ & $2.7 \pm 1.1$ & $2.4 \pm 1.1$ & 0.1 \\
\hline
\end{tabular}

Table 8. Comparison of breast cancer stages based on EORTC QLQ-C30 scale scores

\begin{tabular}{|c|c|c|c|c|}
\hline EORTC QLQ-30 & $\begin{array}{c}\text { STAGE } 1 \\
\text { n: } 53 \\
\text { Mean } \pm \\
\text { SD }\end{array}$ & $\begin{array}{c}\text { STAGE } 2 \\
\text { n: } 128 \\
\text { Mean士 } \\
\text { SD }\end{array}$ & $\begin{array}{c}\text { STAGE } 3 \\
\text { n: } 69 \\
\text { Mean } \pm \\
\text { SD }\end{array}$ & $\begin{array}{c}\mathbf{P} \\
\text { value }\end{array}$ \\
\hline \multicolumn{5}{|l|}{ GLOBAL HEALTH STATE } \\
\hline General Well-being & $9.3 \pm 2.2$ & $9.3 \pm 2.9$ & $8.9 \pm 2 . .9$ & 0.6 \\
\hline \multicolumn{5}{|l|}{ FUNCTIONAL SCALES } \\
\hline Physical functioning & $8.8 \pm 2.7$ & $8.6 \pm 2.9$ & $9.5 \pm 3.0$ & 0.08 \\
\hline Role functioning & $3.5 \pm 1.2$ & $3.6 \pm 1.4$ & $3.8 \pm 1.7$ & 0.5 \\
\hline Cognitive functioning & $3.4 \pm 1.2$ & $3.5 \pm 1.4$ & $3.8 \pm 1.7$ & 0.2 \\
\hline Emotional functioning & $8.1 \pm 2.4$ & $8.2 \pm 3.2$ & $9.2 \pm 3.4$ & 0.06 \\
\hline Social functioning & $4.1 \pm 2.0$ & $4.4 \pm 1.7$ & $4.6 \pm 1.9$ & 0.3 \\
\hline \multicolumn{5}{|l|}{ SYMPTOM SCALES } \\
\hline Dyspnea & $1.4 \pm 0.5$ & $1.5 \pm 0.6$ & $1.7 \pm 0.8$ & 0.08 \\
\hline Nausea-vomiting & $2.8 \pm 1.3$ & $2.9 \pm 1.3$ & $3.0 \pm 1.4$ & 0.6 \\
\hline Loss of appetite & $1.5 \pm 0.6$ & $1.6 \pm 0.8$ & $1.8 \pm 0.8$ & 0.1 \\
\hline Sleep disorders & $2.2 \pm 0.9$ & $2.1 \pm 0.9$ & $2.3 \pm 1.0$ & 0.5 \\
\hline Pain & $3.7 \pm 1.2$ & $3.6 \pm 1.4$ & $4.1 \pm 1.6$ & 0.07 \\
\hline Fatigue & $6.1 \pm 1.7$ & $6.2 \pm 2.4$ & $6.8 \pm 2.5$ & 0.2 \\
\hline Constipation & $1.6 \pm 0.8$ & $1.5 \pm 0.8$ & $1.8 \pm 0.9$ & 0.3 \\
\hline Diarrhea & $1.1 \pm 0.3$ & $1.3 \pm 0.6$ & $1.4 \pm 0.6$ & 0.05 \\
\hline Financial difficulties & $2.0 \pm 1.0$ & $2.5 \pm 1.0$ & $2.7 \pm 1.0$ & 0.007 \\
\hline
\end{tabular}

provements in sexual life $(\mathrm{p}<0.04)$, future perspectives $(\mathrm{p}<0.008)$, and other parameters in stage 1 , without any statistically significant difference between groups ( $p>0.05)$ (Table 9). 


\section{Table 9. Comparison of breast cancer stages based on EORTC QLQ-BR23 scale scores}

\begin{tabular}{|c|c|c|c|c|}
\hline \multirow[b]{2}{*}{ EORTC QLQ-BR23 } & $\begin{array}{c}\text { STAGE } 1 \\
\text { n: } 53\end{array}$ & $\begin{array}{c}\text { STAGE } 2 \\
\text { n: } 128\end{array}$ & $\begin{array}{c}\text { STAGE } 3 \\
\text { n: } 69\end{array}$ & \multirow[b]{2}{*}{$\begin{array}{c}P \\
\text { value }\end{array}$} \\
\hline & $\begin{array}{c}\text { Mean } \pm \\
\text { SD }\end{array}$ & $\begin{array}{l}\text { Mean } \pm \\
\text { SD }\end{array}$ & $\begin{array}{l}\text { Meant } \\
\text { SD }\end{array}$ & \\
\hline \multicolumn{5}{|l|}{ FUNCTIONAL SCALES } \\
\hline Body image & $9.5 \pm 3.6$ & $9.8 \pm 3.6$ & $10.8 \pm 3.9$ & 0.1 \\
\hline Sexual life & $2.7 \pm 1.0$ & $3.3 \pm 1.3$ & $3.0 \pm 1.1$ & 0.04 \\
\hline Sexual pleasure & $1.5 \pm 0.7$ & $1.6 \pm 0.7$ & $1.5 \pm 0.7$ & 0.5 \\
\hline Future perspectives & $2.3 \pm 1.1$ & $2.5 \pm 1.0$ & $3.0 \pm 1.1$ & 0.008 \\
\hline \multicolumn{5}{|l|}{ SYMPTOM SCALES } \\
\hline Adverse effects & $13.8 \pm 3.8$ & $13.2 \pm 4.0$ & $14.6 \pm 4.8$ & 0.07 \\
\hline Breast symptoms & $6.9 \pm 1.9$ & $6.6 \pm 2.5$ & $6.8 \pm 2.5$ & 0.7 \\
\hline Arm symptoms & $5.9 \pm 1.8$ & $5.6 \pm 2.0$ & $6.0 \pm 2.1$ & 0.4 \\
\hline Hair loss & $2.51 \pm .1$ & $2.4 \pm 1.1$ & $1.8 \pm 0.5$ & 0.3 \\
\hline
\end{tabular}

EORTC-QLQ: European Organization for Research and Treatment of Cancer quality of life questionnaire, SD: Standard deviation

\section{Discussion and Conclusions}

Numerous studies performed during the last century revived different treatment alternatives in the management of breast cancer. However when selecting appropriate treatment modality, generally basic targets as survival rates are taken into consideration. However, when evaluating treatment responses the effects of the treatment on quality of life should be also considered. Taking the quality of life into account will aid in selecting more suitable treatment alternatives (9).

Scarce number of studies has investigated the impact of surgical treatments performed on quality of life, social, psychological, and sexual lives of patients with breast cancer (10). In these studies, usually effects of various surgical interventions have been stressed. Distinct from other studies, in our study stage of the disease, age groups, and type of the operation performed were also considered.

In our study, EORTC QLQ-C30, and EORTC QLQ-BR23 questionnaire forms were preferred because of availability of easily intelligible, internationally reliable, and validated Turkish version suitable for our study.

Our study included more participants than other studies in the medical literature (11-15). However their distribution among surgical alternatives are not homogenous (MRM:194, SM:29 and BCS:23) which presumably might influence statistical significance of our results.

Similar to literature findings, we noticed that also in our study mostly MRM, and at a lesser extent BCS had been performed $(11,12)$. Parallel with the literature, educational level of BCS patients was higher than the remaining patients (16).

Our patient population consisted of married ( $\mathrm{n}=237 ; 94.8 \%)$, and divorced $(n=9 ; 3.6 \%)$ women. Seven of nine $(78 \%)$ women were divorced after they were diagnosed with breast cancer. In the literature, the percentage of divorced women after diagnosis of breast can- phenomenon by the more conservative and religious construction of Turkish family structure.

In our study, quality of life of the patients who had undergone breast surgery were evaluated by EORTC QLQ-C30 questionnaires, and in all scales (though in some scales the intergroup difference did not reach statistical significance) MRM group was found to be more adversely affected than the BCS group. In a study performed in our country by Zanapalığlu et al. (18) scores of subgroups of well-being, functional state, and symptom control were significantly better in BCS patients in comparison with the MRM group. In a similar study performed by Dubashi et al (11) detected better general health state in the mastectomy group relative to the BPS group.

According to the assessments based on EORTC QLQ-C30 scores, emotional, and cognitive states were found to be improved in patients with BCS when compared with the MRM group in compliance with the literature findings. Omne-Poten et al (19) detected anxiety, and depression in 45, and $49 \%$ of their MRM patients, while corresponding rates in BCS patients were 40 , and $43 \%$, respectively. However, Ganz et al (20) reported that there was no difference between MRM and BCS groups in terms of psychological parameters one year after the operation.

During evaluations based on responses given to EORTC QLQ-C30 questionnaire items, social status was found to be significantly improved among BCS patients compared with the MRM group. In the literature, numerous studies have many times demonstrated that prolonged and tedious breast cancer treatment effects professional and social life of the patients, and families leading to labor loss, social isolation, despair, feelings of burnout, and depression (21-23).

Symptom scales of EORTC QLQ-BR23 questionnaire such as arm edema, hair loss, and breast symptoms are all related to body image. In our study, perceived body image was affected at a lesser extent in the BCS group when compared with the MRM group. Besides, perceived body images of younger and advanced stage cancer patients were adversely effected. Pyszel et al (24) reported that physical, psychological, and social status disorders are more frequent in patients with arm edema, as well as pain, and fatigue.

When patients were evaluated by EORTC QLQ-C30 items according to age groups, it was identified that the middle age group (Group 2) was more adversely affected from breast cancer. Statistically significant differences were found as for subgroups of cognitive state, and financial difficulties. In the financial subgroup, younger (Group 1 ), and in the cognitive functioning subgroup middle-aged group (Group 2) were more adversely affected. However all parameters in the advanced middle-aged group (Group 3) were minimally effected. Any comparable evaluation was not encountered in the literature. Evaluations based on EORTC QLQ-BR23 responses could not reveal any difference among age groups.

When evaluations were based on patients' responses given to EORTC QLQ-C30 items, and stages of their disease, all parameters of stage 3 patients were more deeply deteriorated. This phenomenon can be explained by more invasive nature of the disease, choice of mastectomy, and radiotherapy, and relatively higher doses of chemotherapy received by the patients. Similar assessments were not encountered in the literature. When evaluations were based on responses given to 
EORTC QLQ-BR23 items, any difference was not detected between disease stages.

In our study, we also questioned post-mastectomy alterations in sexual life. We observed postoperative changes in sexual life less frequently in the BCS group as compared to the MRM group, without any statistically significant intergroup differences. Waldmann et al. (16) also noted that sexual life of the mastectomy group was more unfavorably affected than the BCS group. Zanapalıogluet al.(18) demonstrated that invasive surgical treatment modalities had a profound impact on psychosexual life of Turkish women. Burwell et al. (25) attributed post-mastectomy sexual problems to the deterioration of body image, and decrease sexual desire. Whelan et al. (23) stated that sexual life parameters of patients who had or had not received radiotherapy were similar after 2 years of follow-up period. However, Arora et al. (26) detected relatively lower levels of sexual satisfaction among patients on chemotherapy. Ganz et al. (20) reported higher rates of psychosexual disorders in amenorrheic patients because of chemotherapy.

Changes in sexual life did not differ between age groups. However, sexual lives of the patients with advanced stage (stage 3) disease were deteriorated more significantly. Comparable assessments were not encountered in the literature.

In conclusion; breast cancer is a multi-dimensional disease that induces important derangements in the physical, psychological, and social aspects of the individual's life, and it is one of the most traumatic events that might be experienced by a woman during her lifetime. The physicians should inform their patients in detail about treatment alternatives, and they should reach a mutual consensus. The physicians should also include partners of the patients in every kind of training, and counseling sessions. Every supportive and assistive approach aiming at higher quality of life of the patients will resolve future concerns, and result in favorable physical, psychological, and social outcomes.

Ethics Committee Approval: Ethics committee approval was received for this study from the ethics committee of Mersin University Faculty of Medicine.

Informed Consent: Written informed consent was obtained from patients who participated in this study.

Peer-review: Externally peer-reviewed.

Author Contributions: All authors contributed equally during the preparation of this manuscript.

Financial Disclosure: The authors declared that this study has received no financial support.

Conflict of Interest: No conflict of interest was declared by the authors.

\section{References}

1. Harold JB, Jay RH, Monica M. Malignant Tumors Of The Breast. DeVita, Hellman, And Rosenberg's: Cancer; Principles \& Practice of Oncology. 8th Ed. Vol: Two, Philadelphia 2008; pp1606-1654.

2. De Marquiegui A, Huish M. A womans's sexual life after an operation. BMJ 1999; 318:178-181. (PMID: 9888917) [CrossRef]

3. Gregory D, Johnston R, Pratt G, Watts M, Whatmore S. "Quality of Life”. Dictionary of Human Geography (5 ${ }^{\text {th }}$ ed. June 2009) Oxford: WileyBlackwell.
4. Garratt A, Schmidt L, Mackintosh A, Fitzpatrick R. Quality of life measurement: bibliographic study of patient assessed health outcome measures. BMJ 2002; 324:1417. [CrossRef]

5. Babacan A. Meme kanserinde psikososyal sorunlar ve destekleyici girişimler. Meme Sağlığı Dergisi 2006; 2:129-135.

6. Neil W Scott, Peter M Fayers, Neil K Aaronson, Andrew, Bottomley, Alexander de Graeff, Mogens Groenvold, Chad Gundy, Michael Koller, Morten A Petersen, Mirjam AG Sprangers on behalf of the EORTC Quality of Life Group. EORTC QLQ-C30 Reference Values. http:// groups.eortc.be/qol/eortc-qlq-c30.

7. http://www.proqolid.org/instruments/eortc_quality_of_life_questionnaire_breast_cancer_module_eortc_qlq_br23

8. Demirci S, Eser E, Ozsaran Z, Tankisi D, Aras AB, Ozaydemir G, Anacak Y. Validation of the Turkish versions of EORTC QLQ-C30 and BR23 modules in breast cancer patients. Asian Pac J Cancer Prev 2011; 12:1283-1287. (PMID: 21875283)

9. Bozkurt B, Sülü B, Ünal B, Koçer B, Yıldırım O, Karabeyoğlu M, ve ark. Erken evre meme kanserli hastaların SF-36 ile yasam kalitesinin değerlendirilmesi: Ameliyat Tipi Yasam Kalitesini Etkiliyor mu? Klinikler\&Doktor Dergisi 2004; 10:286-291.

10. Güzelant A, Göksel T, Ozkok S, Tasbakan S, Aysan T, Bottomley A. The European Organisation for Research and Treatment of Cancer C-30: An examination into the cultural validity and reliability of Turkish version of the EORTC QLQ-C30. Eur J Cancer Care 2004, 13:135-144. (PMID: 15115469) [CrossRef]

11. Dubashi B, Vidhubala E, Cyriac S, Sagar TG. Quality of life among younger women with breast cancer: study from a tertiary cancer institute in south India. Indian J Cancer 2010; 47:142-147. (PMID: 20448376) [CrossRef]

12. Salonen P, Kellokumpu-Lehtinen PL, Tarkka MT, Koivisto AM, Kaunonen $\mathrm{M}$. Changes in quality of life in patients with breast cancer. J Clin Nurs 2011; 20:255-266. (PMID: 21108675) [CrossRef]

13. Lee TS, Kilbreath SL, Refshauge KM, Pendlebury SC, Beith JM, Lee MJ. Quality of life of women treated with radiotherapy for breast cancer. Support Care Cancer 2008; 16:399-405. (PMID: 17876610) [CrossRef]

14. Nagel GC, Schmidt S, Strauss BM, Katenkamp D. Quality of life in breast cancer patients: a cluster analytic approach. Empirically derived subgroups of the EORTC-QLQ BR 23--a clinically oriented assessment. Breast Cancer Res Treat 2001;68:75-87. (PMID: 11678311) [CrossRef]

15. Han J, Grothuesmann D, Neises M, Hille U, Hillemanns P. Quality of life and satisfaction after breast cancer operation. Arch Gynecol Obstet 2010; 282:75-82. (PMID: 19960349) [CrossRef]

16. Waldmann A, Pritzkuleit R, Raspe H, Katalinic A. The OVIS study: health related quality of life measured by the EORTC QLQ-C30 and -BR23 in German female patients with breast cancer from Schleswig-Holstein. Qual Life Res 2007; 16:767-776. (PMID: 17286196) [CrossRef]

17. Ivanauskiene R, Kregzdyte R, Padaiga Z. Evaluation of health-related quality of life in patients with breast cancer. Medicina (Kaunas) 2010; 46:351-359. (PMID: 20679752)

18. Zanapalıoğlu Y, Atahan K, Gür S, Çökmez S, Tarcan E. Effect of breast conserving surgery in quality of life in breast cancer patients. J Breast Health 2009; 5: 152-156.

19. Omne-Ponten M, Holmberg L, Burns T, Adami HO, Bergstrom R. Determinants of the psychosocial outcome after operation for breast cancer: Results of a prospective comparative interview study following mastectomy and breast conservation. Eur J Cancer 1992; 28:1062- 1067. [CrossRef]

20. Ganz PA, Schag AC, Lee JJ, Polinsky ML, Tan SJ. Breast conservation versus mastectomy. Is there a difference in psychological adjustment or quality of life in the year after surgery? Cancer 1992; 69:1729-1738. (PMID: 1551058) [CrossRef]

21. Lasry JC, Margolese RG, Poisson R, Shibata H, Fleischer D, Lafleur D, Legault $S$, Taillefer $\mathrm{S}$. Depression and body image following mastectomy and lumpectomy. J Chronic Dis 1987; 40:529-534. (PMID: 3597656) [CrossRef]

22. Onen Sertöz O, Elbi Mete H, Noyan A, Alper M, Kapkaç M. Effects of surgery type on body image, sexuality, self-esteem, and marital adjust- 
ment in breast cancer: a controlled study. Turk Psikiyatri Derg 2004; 15:264-275. (PMID: 15622506)

23. Whelan TJ, Levine M, Julian J, Kirkbride P, Skingley P. The effects of radiation therapy on quality of life of women with breast carcinoma: results of a randomized trial. Ontario Clinical Oncology Group. Cancer 2000; 88:2260-2266. (PMID: 10820347) [CrossRef]

24. Pyszel A, Malyszczak K, Pyszel K, Andrzejak R, Szuba A. Disability, psychological distress and quality of life in breast cancer survivors with arm lymphedema. Lymphology 2006; 39:185-192. (PMID: 17319631)

25. Burwell SR, Case LD, Kaelin C, Avis NE. Sexual problems in younger women after breast cancer surgery. J Clin Oncol 2006; 24:2815-2821. (PMID: 16782919) [CrossRef]

26. Arora NK, Gustafson DH, Hawkins RP, McTavish F, Cella DF, Pingree $\mathrm{S}$, Mendenhall JH, Mahvi DM Impact of surgery and chemotherapy on the quality of life of younger women with breast carcinoma: a prospective study. Cancer 2001; 92:1288-1298. (PMID: 11571745) [CrossRef] 